\title{
Analysis of superplasticizers used in concrete
}

\author{
R.J. Flatt ${ }^{1}$, Y.F. Houst ${ }^{1}$, R. Oesch ${ }^{1}$, P. Bowen ${ }^{1}$, H. Hofmann ${ }^{1}$, J. Widmer ${ }^{2}$, U. Sulser ${ }^{2}$, U. Maeder ${ }^{2}$ \\ and T.A. Bürge ${ }^{2}$
}

\author{
${ }^{1}$ Powder Technology Laboratory, Department of Materials Science, Swiss Federal Institute of Technology, CH-1015 Lausanne, \\ Switzerland \\ ${ }^{2}$ Sika A.G., CH-8048 Zürich, Switzerland
}

\begin{abstract}
Organic polymers are largely used in the processing of ceramic powders in order to improve rheological properties. The industry of hydraulic concrete (initially concretes are also powders) currently uses admixtures called superplasticizers, which allow the production of high performance concrete. Although these admixtures are widely used, their mechanisms of action remain unclear. Measuring the adsorption of these polymers onto the surface of cement particles in aqueous suspensions is usually the first step in related studies. It is often based on a global analysis of the admixture in the aqueous phase, usually by colorimetry or total organic carbon analysis. This traditional approach totally neglects effects linked to molecular mass distribution.

In this paper, we show how HPLC is able to highlight the effect of molecular mass distribution on the adsorption of polycarboxylate-polysulphonate polymers. Measurements were performed with two cements having the same chemical composition and the same specific surfaces, but one was ground with and the other without a grinding aid. It is also shown how adsorption changes as a function of cement hydration.
\end{abstract}

oncrete is a composite material in which aggregates (gravel and sand) are bound by hydrated cement paste. The amount of water required for the reaction of hydration represents about $25 \%$ of the mass of cement. However, in order to obtain a flowing concrete, which can be either cast or pumped, much more water is required (about double). In the long term, this excess water evaporates, leaving voids in the concrete. The associated porosity decreases both the mechanical strength and the durability, and such concrete does not reach the optimal properties it could have if it could be produced as a more compact material.

This is where superplasticizers, also known as High Range Water Reducers (HRWRs), are of interest. Superplasticizers are polymeric dispersants, which when added in small amounts to concrete (typically less than $0.5 \%$ of the mass of cement), allow high water reduction for the same workability. The use of superplasticizers will still grow because of their ability to allow the production of concrete exhibiting high durability. Nowadays, superplasticizers are most widely used in Japan, where it is estimated that almost no concrete is produced without superplasticizers.
Superplasticizers are not only of interest in the concrete industry. They have a much larger scale of application, particularly in ceramic processing. Traditional ceramics (whitewear, sanitary appliances, insulators, etc.), refractories (crucibles, engine components, supports for catalysts, etc.), bioceramics, biocements, watchmaking (watch bodies) are some of the examples of products which include the use of superplasticizers in their production scheme.

The effect of superplasticizers is linked to a change in interparticle forces [1,2]. Defloculation and/or dispersion are terms often used to describe these phenomena. However, a detailed understanding of the underlying mechanisms has not yet been reached. Whether the observed effect is due to electrostatic, steric, entropic or capillary effects or any combination of these, is still a matter of much debate [3-7]. However, a recent generation of superplasticizers appears to act mainly through steric repulsion [6,7]. Despite increased interest in this field, the current knowledge seems insufficient to explain rationally the incompatibilities observed between some superplasticizers and some cements [3, 8-9]. In such cases, it appears that the effect that superplasticizers have on the initial hydration reactions could play a dominant role [8,9], although the influence of the crystalline phase of the aluminate phases has still to be clarified.

When intending to determine either the nature of the dispersive force or its magnitude, it is important to define the extent to which polymers adsorb onto cement particles. So far, most studies in this field have been based on a global analysis of the polymer in solution by either Total Organic Content (TOC) or UV absorption. Adsorbed amounts are then the difference between the initial amount and the amount determined after contacting cement with the solution.

Such approaches totally neglect the effect of polydispersity on adsorption. However, HPLC allows the separation and quantification of various fractions of a polydispersed polymer. GPC on the other hand due to its inherent low resolution is not able to separate the various polymeric fractions [10]. Such low performances are inherent to GPC, as discussed by Nguyen [11] in this issue of Analusis.

If GPC is to be used, a better approach is to fractionate the polymer. Interesting results were obtained in this way by Zhor and Bremmer [12]. They have studied lignosulphonates, a waste product of the paper industry. As a waste product, lignosulphonates do not have a well determined and constant composition. It is therefore important to acquire sufficient knowledge to improve the use of these products as low cost superplasticizers [12,13]. Nevertheless, it is important to be able to analyse in a mix, how the different fractions adsorb. 
Costa et al. [14] showed by HPLC that the extent of polynaphthalene sulphonate (PNS) adsorption onto cement depends on the polymer fraction. Piotte et al. [15] carried out an extensive characterisation of commercial PNS polymers using HPLC and ultrafiltration. They found that the polydispersity of these materials could be as large as 20 . These results clearly show the insufficiency of traditional techniques when discussing dispersion ability with respect to adsorption.

In this paper, HPLC is used to evaluate the effect of polydispersity on the adsorption of polycarboxylate and polysulfonate polymers onto cement. Two cements obtained from the same clinker are studied. One has been ground in presence of a grinding aid (an organic molecule added at a couple ppm) and the other without. Results highlight the importance of using HPLC in adsorption studies.

\section{Experimental}

\section{Cements}

Two Ordinary Portland Cements (OPC) obtained from the same clinker (Olten, Switzerland), were used. The first was ground without any grinding aid. The second was ground in presence of a couple ppm of an acetate of triethanol amine, a common grinding aid. Grinding times were adjusted so as to obtain two OPC with the same specific surfaces.

The BET specific surface area of both powders was determined by $\mathrm{N}_{2}$ adsorption (Micromeritics Gemini III 2375). Three samples were measured for each cement. Bulk chemical compositions and specific surfaces of both cements are given in table $\mathrm{I}$.

\section{Polymers}

Two polycarboxylate-polysulphonate produced as laboratory test products by Sika A.G. (Zürich, Switzerland) were used for this study. Information as to their composition is given in table II. Both are detectable by UV, which allows their adsorption to be measured as described below. Chromatograms of these polymers (Fig. 1 and Fig. 2) show that PCA-2 is relatively monodispersed, while the polymeric fraction of the PCA-1 clearly contains different components. They are supplied as concentrated aqueous solutions, with a solid content of about $30-40 \%$ by mass.

\section{Adsorption}

Adsorption was determined by solution depletion, using HPLC to quantify polymer concentrations. Suspensions of $10 \mathrm{~g}$ cement in $20 \mathrm{~mL}$ demineralised water (conductivity $<0.05 \mu \mathrm{S}$ ) contained in closed polyethylene flasks were placed in a rotary bath $\left(200 \mathrm{rpm}, 25^{\circ} \mathrm{C}\right)$. In a first set of experiments, the polymer was predissolved in the water. In a second set of experiments, polymers were added as concentrated solutions $(10-15 \%$ solid content by mass $)$ with a microdispenser after $30 \mathrm{~min}$ hydration and weighed. The concentration range of the added polymer solution has a viscosity small enough to obtain a precise dosage with microdispensers, while keeping added volumes sufficiently small so as not to change the systems behaviour by dilution. Nevertheless, the dilution was taken into consideration when
Table I. Characteristics of studied cements ${ }^{1}$.

\begin{tabular}{ccc}
\hline & $\begin{array}{c}\text { Cement 1 } \\
\text { With Grinding Aid }\end{array}$ & $\begin{array}{c}\text { Cement } 0 \\
\text { Without Grinding Aid }\end{array}$ \\
\hline $\mathrm{LOl}\left(100 \mathrm{CO}^{\circ} \mathrm{C}\right)$ & 2.89 & 2.65 \\
$\mathrm{CO}_{2}$ & 2.61 & 2.29 \\
$\mathrm{SiO}_{2}$ & 20.35 & 20.51 \\
$\mathrm{Al}_{2} \mathrm{O}_{3}$ & 5.26 & 5.28 \\
$\mathrm{Fe}_{2} \mathrm{O}_{3}$ & 2.92 & 2.91 \\
$\mathrm{CaO}_{\mathrm{SO}}$ & 63.19 & 63.18 \\
$\mathrm{MgO}$ & 2.25 & 2.20 \\
$\mathrm{~K}_{2} \mathrm{O}$ & 1.52 & 1.58 \\
$\mathrm{Na}_{2} \mathrm{O}$ & 1.10 & 1.11 \\
$\mathrm{MnO}_{\mathrm{TiO}}$ & 0.26 & 0.28 \\
$\mathrm{CaO}$ (free) & 0.05 & 0.05 \\
Specific Surface & 0.24 & 0.25 \\
Blaine $\left[\mathrm{m}^{2} \mathrm{~g}^{-1}\right]$ & 0.07 & 0.07 \\
Specific Surface & & \\
BET $\left[\mathrm{m}^{2} \mathrm{~g}^{-1}\right]^{2}$ & $0.96 \pm 0.09$ & 0.349 \\
\hline
\end{tabular}

1 Chemical composition were determined by the Institut für Baustofflehre und Materialprüfung of the University of Innsbruck. Blaine Specific Surface was determined by ZAG, the Slovenian Center for Materials evalutation in Lublijana.

2 Values are an average of three measurements. Errors given correspond to the $95 \%$ confidence interval.

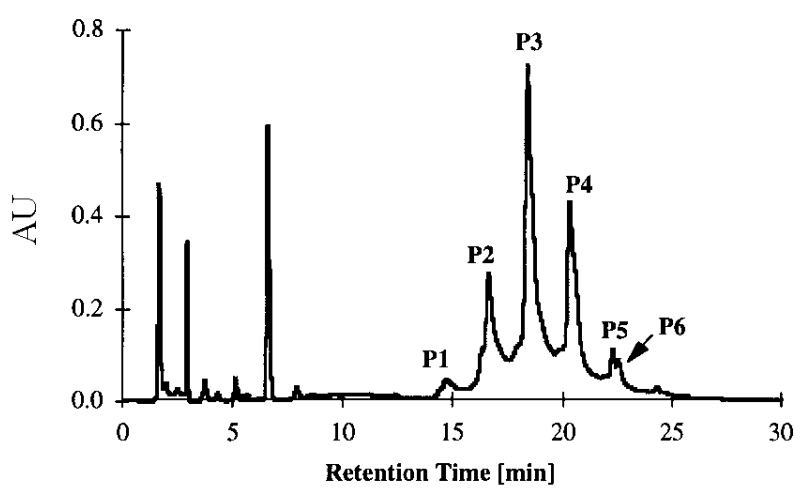

Figure 1. HPLC Chromatogram for polymer PCA-1.

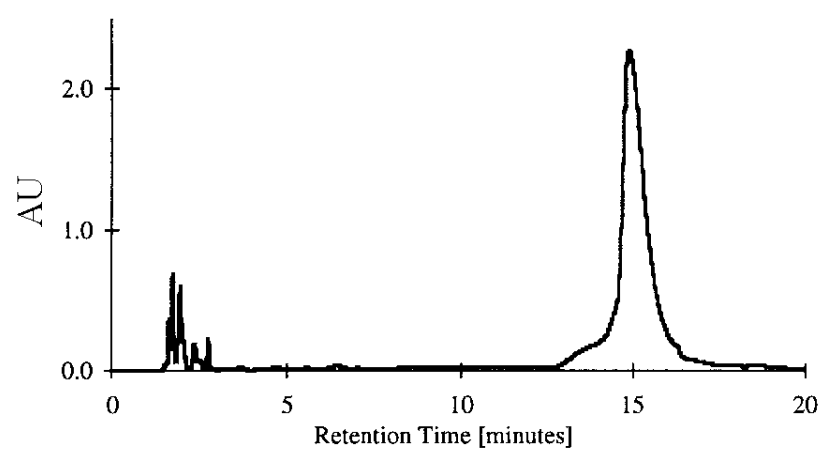

Figure 2. HPLC Chromatogram for PCA-2. 
Table II. Characteristics of the polymers used.

\begin{tabular}{ccccc} 
Designation & Chemical Type & $\begin{array}{c}\text { Number Average } \\
\text { Molecular Mass } \\
{\left[\mathrm{g} \mathrm{mol} \mathrm{m}^{-1}\right]}\end{array}$ & $\begin{array}{c}\text { Mass Average } \\
\text { Molecular Mass } \\
{\left[\mathrm{g} \mathrm{mol} \mathrm{mol}^{-1}\right]}\end{array}$ & $\begin{array}{c}\text { Polydispersity } \\
\text { Coefficient } \\
\mathrm{Mw} / \mathrm{Mn}[-]\end{array}$ \\
\hline PCA-1 & $\begin{array}{c}\text { Na-polycarboxylate- } \\
\text { polysulfonate }\end{array}$ & $15600 \pm 2000$ & $40500 \pm 5000$ & 2.6 \\
PCA-2 & $\begin{array}{c}\text { Na-polycarboxylate- } \\
\text { polysulfonate }\end{array}$ & $11500 \pm 2000$ & $24500 \pm 5000$ & 2.1 \\
\hline
\end{tabular}

calculating the adsorption mass balance. Adsorption time was $30 \mathrm{~min}$, which was found to be sufficient to reach almost complete surface coverage. Large adsorption times were not investigated because previous test had shown that excessive residence of the polymers in an alkaline solution could lead to a partial hydrolysis and degradation.

After adsorption, the suspensions were centrifuged ( $5 \mathrm{~min}, 3000 \mathrm{rpm}, 25^{\circ} \mathrm{C}$ ) in a thermostated table centrifuge from Jouan (mod. CR-412; St-Nazaire, France). From the supernatant, $5 \mathrm{~mL}$, buffered with $30 \mu \mathrm{L}$ of $\mathrm{CH}_{3} \mathrm{COOH}$ (Merck; analysis quality), were filtered through a $0.45 \mu \mathrm{m}$ filter mounted on a disposable syringe. The first $2 \mathrm{~mL}$ were discarded and the tests was analysed by reverse phase chromatography with a high performance liquid chromatograph from Waters (mod. Alliance; Milford, USA) equipped with a diode array detector. The column was an RP-18 from Merck. A gradient between $\mathrm{H}_{2} \mathrm{SO}_{4} 0.01 \mathrm{~N}$ and acetonitrile was used to elute the samples. Each sample was analysed three times.

Because dissolved impurities from cement can alter the polymers, standards were not prepared in pure water. Instead, they were prepared in solutions having the same composition as the liquid phase of the cement suspensions. These were obtained as follows. Conductivity measurements showed that, after less than $10 \mathrm{~min}$, the liquid phase was stable. Large volumes of suspensions have the same solid/liquid ratio were mixed $10 \mathrm{~min}$ and then centrifuged and filtered $(0.2 \mu \mathrm{m})$. The solutions obtained were kept in closed bottles. Large storage times were avoided because of the risk of carbonating the solution. These calibration samples, apart from containing no powder and not being centrifuged, were treated in the same way as the other samples.

\section{Results}

\section{General trends}

The common procedure to determine adsorption from solution depletion, is described hereafter. The responses (peak height or surface) of a series of samples are correlated with the known concentrations of these same samples. A calibration curve is then obtained by plotting concentrations versus response, as in figure 3 (filled diamonds). In this case, a straight line through the origin is obtained.

The concentrations of polymers remaining in the liquid phase of a suspension can then be determined. The amounts of adsorbed polymers are obtained by taking the difference

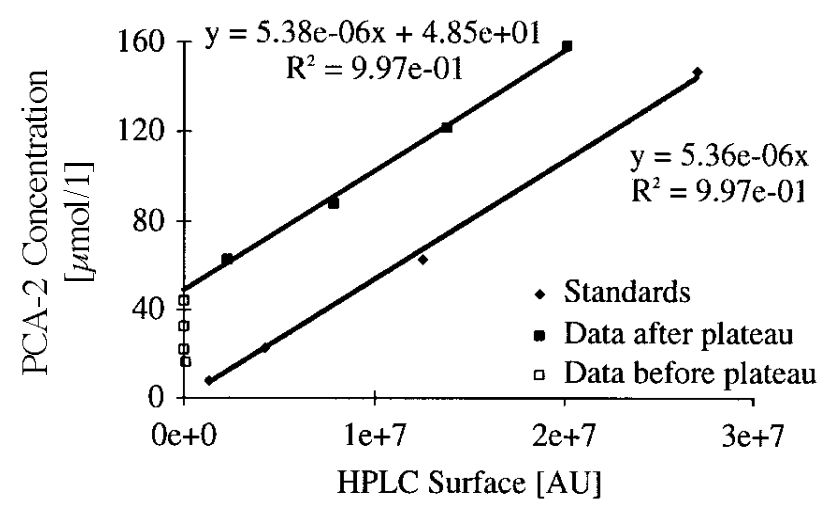

Figure 3. Adsorption raw data for polymer PCA-1 and cement ground with a grinding aid.

between amounts introduced into the suspension and those analysed in the liquid phase after a given time. These values are given with respect to the amount of powder (mass or surface) in the suspensions and plotted versus the concentration of polymers remaining in solution.

When polymer PCA-2 is contacted with $\mathrm{MgO}$, a model powder for cement, adsorption exhibits a step like function $[16,17]$. This means that until the surface is saturated, all the polymers present in the suspensions are adsorbed onto surface of the particles. The concentrations of polymers at the surface of these particles which correspond to saturated surfaces will be referred to as the TASC, the Total Adsorbable Superplasticizer Concentration [18]. Polymers in excess of this amount remain polymers in the liquid phases. From the analytical point of view, this means that no responses are measured for solutions having an initial concentration below the TASC, while above it responses are measured.

In addition to calibration data, figure 3 shows adsorption of PCA-2 in a suspension of cement 1 (With Grinding Aid) when the polymer is added after 30 min hydration. The open squares represent points below the TASC. No responses are measured in the final liquid phases and all abscissa are equal to zero. The filled squares represent points above the TASC. Their abscissa are greater than zero. In this figure, the slope obtained from the linear regression over these data (filled squares) appears identical to the one obtained from the standards (filled diamonds). Indeed, it turns out that the confidence with which one could reject the hypothesis that both slopes are equal is only 3\%. Therefore, experimental data does not allow to differentiate both slopes and these may reasonably be considered as equal. It is important to note 
that this behaviour is a consequence of the stepped like function of PCA-2 adsorption.

Surface saturation concentrations or TASC values can therefore be obtained without using calibration curves. It is sufficient to take the ordinate at the origin from the linear regression performed over adsorption data for which responses are greater than zero (Fig. 3). This requires all suspensions to have almost identical masses, which was the case in all experiments (largest mass: $10.016 \mathrm{~g}$, lowest mass: $9.992 \mathrm{~g}$ ).

\section{PCA-1}

Polymer PCA-1 adsorbs in a similar way to PCA-2. However, its polydispersity $(\mathrm{Mw} / \mathrm{Mn})$ is larger and its polymeric fraction is separated into 6 different peaks instead (Fig. 1) of one (Fig. 2). These peaks may be due either to fractions of different masses and/or conformations. These fractions appear to adsorb with step like functions. Their TASC values and corresponding errors are determined, as described above, by linear regressions. In this case however, peak heights were used instead of peak surfaces because they provide better correlations for both standards and adsorption samples. The reason behind this, is that the chromatographic method cannot totally separate these fractions and that surfaces are partly overlapping.

TASC values for the various polymeric fractions of PCA-1 have the following physical meaning. They define the concentrations of added superplasticizer beyond which a given fraction stops adsorbing. Though initially all fractions adsorb, it is shown further that they do not all stop adsorbing at the same amount of added polymer. Thus, there is a range over which only some fractions of the added polymer are still adsorbing. In what follows, adsorption of the polymeric fractions of PCA-1 are considered with respect to cements 0 and 1 (without and with grinding aid) and with respect to hydration times 0 and 30 minutes.

Since the specific surfaces between both cements cannot be considered different (confidence interval for rejecting equality is at most $60 \%$ ), adsorbed concentrations are given with respect to the mass of cement in the suspensions. Values with respect to surface area are almost the same since these surfaces are very close to $1 \mathrm{~m}^{2} \mathrm{~g}^{-1}$.

\section{Effect of polymeric fraction}

Figure 4 shows adsorption data for both cements when polymer PCA-1 is contained in the mixing water (0 minute hydration). All TASC values along with error bars corresponding to $90 \%$ confidence intervals are plotted against retention time. It appears that the polymeric fractions with larger retention times might have lower TASC values. However, it is difficult from such a graph to decide whether this trend is or not the result of experimental errors. This problem is even greater in figure 5, which shows the case where superplasticizers are added after 30 min hydration.

In order to answer the above question, one tests whether the slope of TASC values versus retention time is equal to zero $\left(m_{\mathrm{rt}, \mathrm{ads}}=0\right.$ ?). If $m_{\mathrm{rt}, \text { ads }} \neq 0$, retention time is correlated with a change in TASC.

Because all TASC values have different errors, they should not be weighted in the same way when calculating

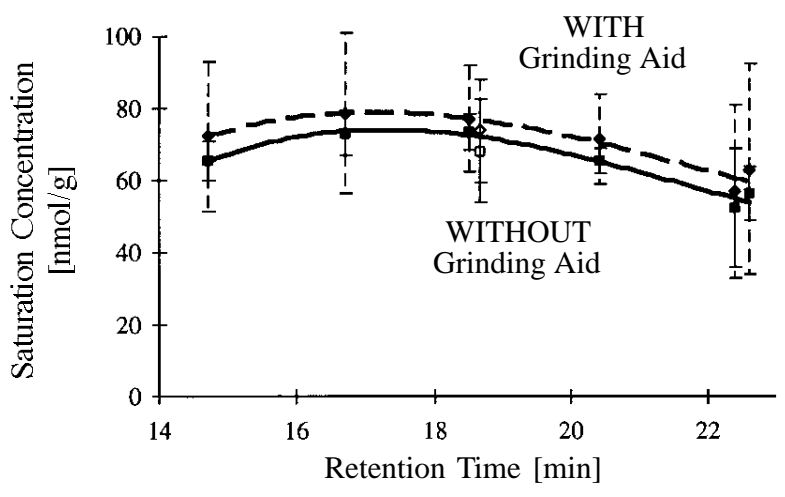

Figure 4. TASC values for polymeric fractions of PCA-1 as a function of retention times. Results are given for both cements and hydration time of $0 \mathrm{~min}$. Error bars correspond to $90 \%$ confidence intervals. Filled symbols refer to fractions and empty symbols to the overall polymer.

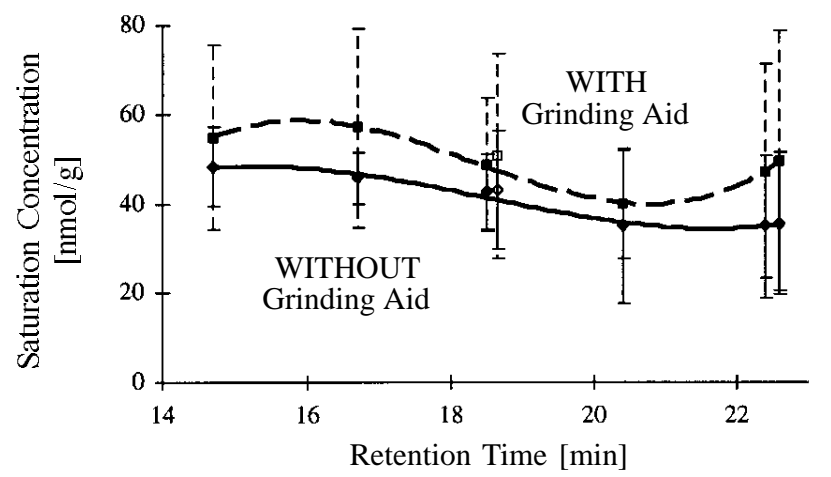

Figure 5. TASC values for polymeric fractions of PCA-1 as a function of retention times. Results are given both cements and hydration time of $\mathbf{3 0} \mathrm{min}$. Error bars correspond to $90 \%$ confidence intervals. Filled symbols refer to fractions and empty symbols to the overall polymer.

$m_{\mathrm{rt}, \mathrm{ads}}$. Weights are taken as the inverse of the standard deviation of each point. After normalising $m_{\mathrm{rt}, \mathrm{ads}}$ by its standard deviation, it is possible to determine from a Student law $t_{(1-\alpha ; n-2)}$, the confidence level $\alpha$ up to which one can reject $m_{\mathrm{rtads}}=0$. In this test, the degree of freedom is $n-2$, where $n$ is the number of retention times considered.

These degrees of confidence are shown in table III. Values for cement 0 (without grinding aid) are high. In this case it is possible to consider that retention time is correlated with TASC. In other words, the polymerised part of superplasticizer PCA-1 contains fractions (different mass and/or conformation) which do not all adsorb in the same way. On the other hand, values for cement 1 (with grinding aid) are lower. In this case, the correlation might exist but if it does, its effect is too low to be determined within the range of experimental errors.

\section{Effect of hydration time}

For each cement, the effect of hydration time on adsorption is tested by comparing TASC values obtained after 0 and 30 min hydration, for each polymeric fraction. Table IV gives the degrees of confidence up to which 
Table III. Confidence intervals for rejecting the absence of a correlation between the amount of PCA-1 for which a given fraction reaches surface saturation and the retention time of these fractions.

\begin{tabular}{ccc} 
& Cement 0 & Cement 1 \\
\hline Hydration: 0 min & $99 \%$ & $92 \%$ \\
Hydration: 30 min & $97 \%$ & $87 \%$ \\
\hline
\end{tabular}

TASC $_{0 \text { min, cement } x \text {, fraction } i}=\operatorname{TASC}_{30 \text { min, cement } x \text {, fraction } i}$ can be rejected.

The correct way to obtain these values is described in [19]. Results indicate that hydration decreases the amount of adsorbed polymers in a significant way for both cements. A comparison of the effects of hydration can be seen in figure 6 for cement 0 and in figure 7 for cement 1 .

\section{Effect of grinding aid}

For each hydration time, the effect of a grinding aid on adsorption is tested by comparing TASC values obtained with cement 0 and cement 1 . The degrees of confidence up to which TASC cement $0, x \min$, fraction $i=$ TASC $_{\text {cement } 1, x \text { min, fraction } i}$ can be rejected are given in table V. Values are low and it is concluded that if grinding aid influences adsorption its effect cannot be detected within the range of experimental errors. The effect of grinding aid can be seen in figure 4 for 0 minute hydration and in figure 5 for 30 minutes hydration.

\section{Discussion}

Polymer PCA-2 underwent a slight micellisation. The solutions had been obtained by extraction from suspensions having the same liquid/solid ratios as the adsorption samples. This was done in order to obtain solutions with ionic compositions very similar to the aqueous phases of the cement suspensions. Micellisation is indicated by the appearance of small peaks at larger retention time than in figure 1 . It is most likely the trivalent aluminium ions which are responsible for this. The same effect is indeed seen in suspensions of $\mathrm{MgO} \cdot \mathrm{Al}_{2} \mathrm{O}_{3}$ but not in suspensions of $\mathrm{MgO}$ or $\mathrm{Mg}(\mathrm{OH})_{2}$. However, these peaks are small and are present in both the adsorption and standard samples. This effect was not observed with PCA-1.

\section{Effect of polymeric fraction}

Polymer PCA-1 is a test product which presents good superplasticizing properties. However, as is apparent in its chromatogram (Fig. 1), it contains some products of low mole-

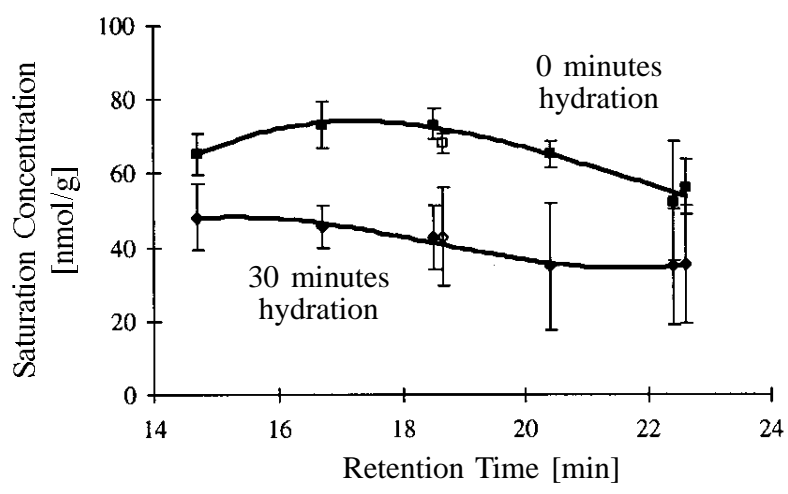

Figure 6. TASC values for polymeric fractions of PCA-1 as a function of retention times. Results are given both cement without grinding aid for hydration times of $\mathbf{0}$ and $\mathbf{3 0} \mathrm{min}$. Error bars correspond to $90 \%$ confidence intervals. Filled symbols refer to fractions and empty symbols to the overall polymer.

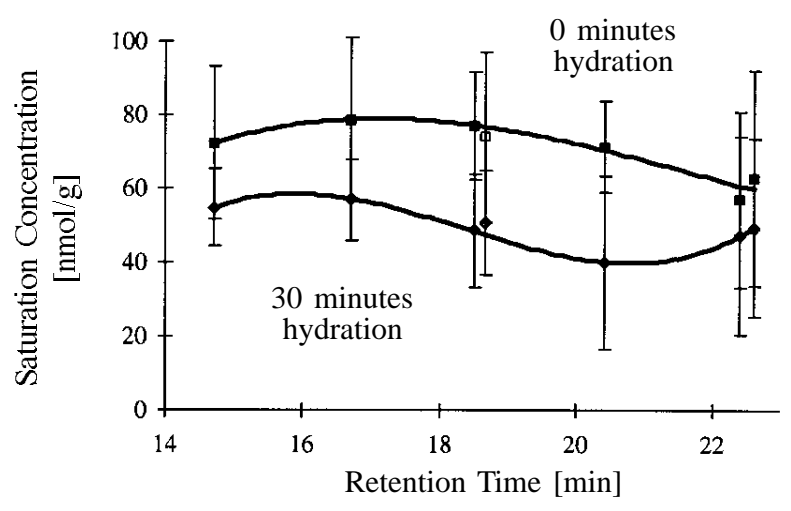

Figure 7. TASC values for polymeric fractions of PCA-1 as a function of retention times. Results are given for both cement with grinding aid for hydration times of $\mathbf{0}$ and $\mathbf{3 0} \mathrm{min}$. Error bars correspond to $90 \%$ confidence intervals. Filled symbols refer to fractions and empty symbols to the overall polymer.

cular weight (those at very low retention times). For cost reasons, no separation is done. Because these were not found to adsorb significantly, they are not expected to alter the superplasticizer performance. The very low molecular mass products (small retention times) do not have sufficiently strong interactions to adsorb (their adsorption energy would be lower than thermal energy $k_{\mathrm{B}} T$ ). On the other hand, the polymer fractions adsorb onto the cement particle and they are expected to induce the main fluidifying effects.

Table IV. Comparison of the effect of hydration on adsorption onto cements 0 and 1. Confidence intervals for rejecting the absence of an effect of hydration on adsorbed amounts. Values are given for each peak (polymeric fraction). The last value is obtained using the total surface of the polymeric fraction of PCA-1.

\begin{tabular}{cccccccc} 
& peak 1 & peak 2 & peak 3 & peak 4 & peak 5 & peak 6 & Global \\
\hline Cement 0 & $96 \%$ & $100 \%$ & $100 \%$ & $97 \%$ & $75 \%$ & $90 \%$ & $100 \%$ \\
Cement 1 & $69 \%$ & $75 \%$ & $90 \%$ & $87 \%$ & $32 \%$ & $40 \%$ & $84 \%$ \\
\hline
\end{tabular}


Table V. Comparison between cements 0 and 1 at hydration times of 0 and 30 min. Confidence intervals for rejecting the absence of an effect of hydration on adsorbed amounts. Values are given for each peak (polymeric fraction). The last value is obtained using the total surface of the polymeric fraction of PCA-1.

\begin{tabular}{lccccccc}
\hline & peak 1 & peak 2 & peak 3 & peak 4 & peak 5 & peak 6 & Global \\
\hline Hydration (0 min) & $52 \%$ & $39 \%$ & $21 \%$ & $25 \%$ & $15 \%$ & $27 \%$ & $40 \%$ \\
Hydration (30 min) & $43 \%$ & $65 \%$ & $52 \%$ & $40 \%$ & $62 \%$ & $61 \%$ & $56 \%$ \\
\hline
\end{tabular}

In what follows, we discuss the adsorption of the polymeric fraction of PCA-1. Four different situations are considered: two cements (with and without grinding aid), and two modes of adding superplasticizer (in the mixing water or after $30 \mathrm{~min}$ of cement hydration). In order to clarify data analysis, we start with the case of cement 0 when PCA- 1 is included in mixing water. Adsorption of the PCA-1 fractions can be seen in figure 4 as a function of the retention times $(R T)$ of these fractions. The values in this figure correspond, for each PCA-1 fraction, to the amount of added PCA-1 below which all the given fraction is adsorbed. For instance, the fraction with $R T=14.7 \mathrm{~min}(\mathrm{~F} 1)$ is totally adsorbed until more than $73 \mathrm{nmol} / \mathrm{g}$ PCA-1 are added to the suspension. Above this concentration, the excess of this fraction remains in the aqueous phase. Below it, the entire fraction can be adsorbed onto the surface of the cement particles. On the other hand, the fraction with $R T=22.3 \mathrm{~min}$ (F5) is totally adsorbed only until $53 \mathrm{nmol} / \mathrm{g}$ PCA-1 are added. This means that below $53 \mathrm{nmol} / \mathrm{g}$, both $\mathrm{F} 1$ and F5 can be totally adsorbed. Between 54 and $73 \mathrm{nmol} / \mathrm{g}$ of added PCA-1, all F1 can be adsorbed, while an excess of F5 remains in solution. Above $73 \mathrm{nmol} / \mathrm{g}$, both fractions are beyond saturation and their excess remain in the aqueous phase.

However, it is difficult, because of the magnitude of experimental errors to decide whether such differences are statistically significant. In order to solve this uncertainty, we must decide whether retention time and adsorption (TASC) are correlated. This is done by testing the degree of confidence with which one can reject the absence of such a correlation. A high value means there is a correlation. A low value means, that there might be a correlation, but if so, its effect cannot be detected within experimental errors.

When PCA-1 is added in mixing water (Fig. 4), adsorption turns out to be well correlated with retention time (Tab. III, first line). The confidence interval for rejecting the absence of such a correlation is higher for cement $0(99 \%)$ than for cement $1(92 \%)$. Both confidence levels decrease when PCA-1 is added after 30 min hydration (Fig. 5), as indicated in the second line of table III.

A correlation is clear between retention time and adsorption for cement 0 . For cement 1 , this correlation is lower, because errors are larger. The reason why larger errors are obtained with cement 1 is unclear. However, it appears reasonable to conclude that on both cements, adsorption is correlated with retention time. In other words, the fractions with high retention times reach saturation for lower amounts of added PCA-1.

In order to interpret this correlation, it is necessary to relate retention time to a property of the different PCA-1 fractions. With similar HPLC methods, Piotte et al. [11] found, for PNS, that the fractions containing the largest polymers have the largest retention times. For PCA-1, it is also possible that retention times are linked to conformation differences. In this case, the fractions with the largest retention times would have the highest surface interaction with the HPLC column. It is most likely that these same fractions will also require larger surface areas for adsorption onto the surface of cement particles. Both cases may therefore be united and we can consider that larger retention times can be associated with larger surface requirements for adsorption. In turn, this would mean that the fractions having the larger surface requirements per molecule reach saturation for lower amounts of added superplasticizer (lower surface coverages). Up to a certain amount of added superplasticizer, all fractions adsorb. Then, beyond a certain surface coverage, some fraction can no longer adsorb. The fractions with smaller retention times need less space than the fractions with larger retention times. They can still find adsorption sites when the others cannot.

It should be noted that adsorption of PCA-1 fractions are not found to be correlated with initial peak intensities. Therefore, a straight forward kinetic law linked to polymer availability is insufficient to explain adsorption differences.

The correlation between polymer fractions and adsorption shows that a global analysis of polymer adsorption (by spectrometric UV absorption or total organic content) results in a loss of information. This could lead to misinterpretation of data when trying to relate adsorption properties to rheological data.

An effect of polydispersity is seen with PCA-1 which has a relatively low polydispersity coefficient (2.6). Larger effects could therefore be expected with polymers such as PNS or lignosulphonates which usually have relatively large polydispersity coefficients $[12,15]$. For instance, the samples of ultrafiltrated lignosulphonate which perform best in the study by Zhor and Bremmer [12], have dispersities still larger than PCA-1. Separations and better understanding of optimal usage should allow increased performances with lignosulphonates at relatively low cost since (lignosulphonates are a waste product from the paper industry). However, such an approach is not expected to remain cost efficient if performances similar to those of synthetic polymers are required.

\section{Effect of hydration time and of grinding aid}

Superplasticizers can be used either to decrease the amount of required water for a given fluidity or to increase fluidity at a given solid loading. In the first case, superplasticizers are added with the mixing water, while in the second, they are added after a given time as concentrated solutions. Various authors have shown that superplasticizers alter the morphology of early hydrates such as ettringite (cubes instead of needles). This is mainly going to affect the applications of the first category. Therefore, when discussing 
diverse effects of superplasticizers between such systems it is important to introduce chemical dynamics. This also includes considering whether adsorption is different in the fresh and the hydrated cement pastes.

Results shown in figure 6 and figure 7 show that hydration decreases adsorption on both cements. The levels of significance are very high for cement without grinding aid and a little lower for the cement with grinding aid (Tab. IV). This decrease might be due to formation of a hydration layer, which would have less affinity for superplasticizers. It might also be due to the passivation of specific adsorption sites by hydration (probably the aluminate phases).

On the other hand, no significant difference is seen between both cements at either hydration time (Fig. 4, Fig. 5, Tab. V). It is therefore concluded that if grinding aid alters adsorption of PCA-1, its effect is too small to be detected within experimental errors of these measurements. It must be noted that errors are systematically larger for the cement with grinding aid. This decreases both confidence levels when comparing one cement to an other. However, even if an effect due to grinding aid was present, it would remain small.

\section{Conclusions}

In this study, two polymers differing mainly from their molecular mass distributions have been considered. Polymer PCA-2 which has a narrow molecular mass distribution, is used to show how adsorption can be determined provided adsorption follows a step like function. Polymer PCA-1 is used to show the effect of polydispersity on adsorption.

It is found that the various fractions of its polymeric part do not adsorb in the same way. This is an important result, because it shows the importance of using HPLC when determining polymer adsorption onto powders. With PCA-1, overall analysis such as TOC or UV adsorption might lead to data misinterpretation (50\% risk of committing and error larger than $30 \%$ ).

It has been reported that maximum performance of superplasticitzers is often achieved before surface saturation [10, 20-21]. This can be due to the polydispersity of the polymers used. For instance, if steric repulsion is a dominant mechanism, the larger polymers would be expected to be most effective. Completing adsorption of these fractions required to add less PCA-1 than for completing adsorption of the other fractions. This means that if one determines the amount of added polymer required to complete adsorption by an overall analysis of polymer content, the value obtained is larger. Therefore, traditional adsorption methods can lead to overestimating polymer requirements for surface saturation of the most effective polymeric fractions. Comparing such results with rheological data can then lead to believing that the surface does not need to be saturated to reach optimal fluidity, which is precisely what the above mentioned researchers found. In fact, the surface might have to be saturated with the larger and most effective polymeric fractions.

Another factor which should not be underevaluated is of solid loading. We have shown earlier, using model systems, that superplasticizer dosage and solid ratio are not independent parameters [18]. Furthermore, it appears that the maximal solid loading is reached for total surface coverage. Therefore, with higher solid loadings, polymer requirements might well increase for the above mentioned studies.

Hydration is also shown to alter superplasticizer adsorption, while a grinding aid containing triethanolamine acetate does not have a significant effect. Resolving the question of the effect of a grinding aid is important, since it could be a hidden parameter between cements of different origins. It seems that grinding with triethanolamine (a couple ppm) to achieve the same surface does not alter PCA-1 adsorption.

However, significant rheological differences are measured between both cements [22,23]. Such differences remain therefore to be explained more by particle size distributions and chemical dynamics than by DLVO theory applied to monosized spheres.

This paper has focused on the effect that polydispersity can have on polymer adsorption. A topic which is not only of interest for the concrete industry, but also to ceramic processing.

\section{Acknowledgements}

This research was made within the framework of the EUREKA Project 1363 ICHRWR and received financial support from the Swiss Commission for Technology and Innovation (CTI) which is gratefully acknowledged.

\section{References}

1. Horn, R. G. "Surface forces and their Action in Ceramic Materials", J. Amer. Cer. Soc. 1990, 73(5), 1117-1135.

2. Israelachvili, J. "Intermolecular \& Surface Forces", Academic Press Ltd., Suffolk, 1990.

3. Aïtcin, P. -C.; Jolicoeur, C.; MacGregor, J. G.; "Superplasticizers: How They Work and Why They Occasionally Don't", Concrete Intern. 1994, 16(5), 45-52.

4. Sakai, E.; Daimon, M. "Mechanisms of Superplastification", Materials Science of Concrete IV; Skalny J. and Mindess S, Eds., The Amercian Ceramic Society, Westerville, OH, 1995; pp 91-111.

5. Jolicoeur, C.; Nkinamubanzi, P. C.; Simard, M. A.; Piotte, M. "Progress in Understanding the Functional Properties of Superplasticizers in Fresh Concrete", Proceedings of the 4th CANMET/ACI International Conference on Superplasticizers and Other Chemical Admixtures, Malhotra V. M., Ed., American Concrete Institute. SP-148, Detroit, 1994; pp 63-87

6. Sakai, E.; Daimon, M. "Dispersion Mechanisms of Alite Stabilized by Superplasticizers Containing Polyethylene Oxide Graft Chains", Proceedings of the 5th Canmet/ACI International Conference on Superplasticizters and Other Chemical Admixtures in Concrete Malhotra, V. M., Ed., American Concrete Institute, Detroit. 1997; SP-173, pp 187202.

7. Uchikawa, H.; Hanehara, S.; Sawaki, D. "Effect of Electrostatic and Steric Repulsive Force on the Dispersion of Cement Particles in Fresh Cement Paste", Proceedings of the 10th International Congress on the Chemistry of Cement, V. 3, Justness, H. J. Ed., 1997, Paper 3iii001. 
8. Costa, U.; Corazza, F.; Colombet, P.; Fernon, V.; Vichot, A. "Mechanisms of Cement - Admixture Interaction", in Ref. [7], Paper 3 iii003.

9. Fernon, V.; Vichot, A.; Le Goanvic, N.; Colombet, P.; Corazza, F.; Costa, U. "Interaction Between Portland Cement Hydrates and Polynaphtalene Sulpfonates", in Ref. [6], pp 225-248.

10. Ferrari, G.; Cerulli, T.; Clemente, P.; Dragoni, M. "Adsorption of Naphtalene Sulfonate Superplasticizers by Cement Particle Through Gel Permeation Chromatography", in Ref. [6]. SP173, pp 869-892.

11. Ngyuen, T. Q.; Kaush, H. H. «Chromatographie en phase liquide des polymères synthétiques : principes et applications », Analusis, current issue.

12. Zhor, J.; Bremmer, T. W. "Influence of Molecular Weight Fractions on the Properties of Fresh Cement", in Ref. [6], pp 781-805.

13. Coppola, L.; Erali, E.; Troli, R.; Collepardi, M. "Blending of Acrylic Superplasticizer with Naphtelene, Melamine or Lignosulfonate-Based Superplasticizers", in Ref. [6], pp 203-224.

14. Costa, U.; Goisis, M.; Guerra, G. "Application of HPLC in the Characterization of Naphalene Sulphonate-Based Superplasticizers", Proceedings of the 9th International Congress on the Chemistry of Cement, New Delhi, India, 1992; Vol. IV, pp 619-626.

15. Piotte, M.; Bossányi, F.; Perreault, F.; Jolicoeur, C. "Characterization of poly(naphtalenesulfonate) salts by ion- pair chromatography and ultrafiltration", J. Chrom. A, 1995, 704, 377-385.

16. Flatt, R. J.; Houst, Y. F.; Bowen, P.; Hofmann, H.; Widmer, J.; Sulser, U.; Maeder, U.; Bürge, T. A., "Interaction of Superplasticizers With Model Powders in a Highly Alkaline Medium", in Ref. [6], pp 743-762.

17. Flatt, R. J.; Houst, Y. F.; Bowen, P.; Hofmann,H.; Widmer, J.; Sulser, U.; Maeder, U.; Bürge, T. A. "Adsorption of superplasticizers on model powders: temperature dependence, effect on zeta potential and role of chemical structure", in Ref. [7], Paper 3iii002.

18. Flatt, R. J.; Houst, Y. F.; Bowen, P.; Hofmann, H.; Widmer, J.; Sulser, U.; Maeder, U.; Bürge, T. A. "Effect of Superplasticizers in a Highly Alkaline Model Suspensions Containing Silica Fume", Proceedings of the 6th Canmet/ACI International Conference on Superplasticizters and Other Chemical Admixtures in Concrete, Bangkok Thailland 31 June-5 June 1998, accepted.

19. Green, J. R.; Margerison, D. "Physical Sciences Data 2: Statistical Treatment of Experimental Data", Elsevier. Amsterdam. 1978, chapter 14, Sect. 6.5.1, p 322.

20. Matsuyama, H.; Lewis, J. A.; Young, J. F. "Dispersion Mechanism in Processing Cement Paste", Proceedings of the $2^{\text {nd }}$ RILEM Workshop on Hydration and Setting, Dijon France, 11-13 June 1997, submitted.

21. Matsuyama, H. 1997, personal communication.

22. Flatt, R. J.; Houst, Y. F.; Bowen, P.; Hoffmann, H.; Widmer, J.; Sulser, U.; Maeder, U.; Bürge, T. A. work in progress. 